\title{
O-glycosylated pro-B-type natriuretic peptide is cleaved intracellularly by furin in ventricular and atrial myocytes: importance of the distance between the O-glycosylation and cleavage sites
}

\author{
Toshio Nishikimi ${ }^{1,5^{*}}$, Yasuaki Nakagawa ${ }^{1}$, Naoto Minamino ${ }^{4}$, Masashi Ikeda $^{3}$, Toshihiko Ishimitsu ${ }^{2}$, Chinatsu Yamada', \\ Kazuhiro Nakao ${ }^{1}$, Takeya Minami ${ }^{1}$, Yoshihiro Kuwabara ${ }^{1}$, Hideyuki Kinoshita ${ }^{1}$, Koichiro Kuwahara ${ }^{1}$, Kenji Kangawa ${ }^{4}$, \\ Kazuwa Nakao ${ }^{1}$
}

From 6th International Conference on cGMP: Generators, Effectors and Therapeutic Implications Erfurt, Germany. 28-30 June 2013

\section{Background}

Our objective was to investigate the molecular mechanism underlying the processing of proBNP, levels of which are increased in heart failure $[1,2]$.

\section{Methods}

Rat neonatal atrial and ventricular myocytes were cultured separately. We examined: (1) the molecular forms of secreted and intracellular BNP in atrial and ventricular myocytes; (2) levels of corin and furin mRNA in atrial and ventricular myocytes and the effect their knockdown on proBNP processing; (3) molecular forms of BNP in plasma from rats and humans with and without heart failure; (4) the structure of proBNP in humans and rats; (5) the impact of the distance between the glycosylation and cleavage sites in wild-type and mutant human proBNP expressed in rat myocytes transfected with lentiviral vectors.

\section{Results}

BNP was the major molecular form secreted by atrial $(75 \pm 9 \%)$ and ventricular ( $85 \pm 9 \%)$ myocytes, and was the major intracellular form in atrial myocytes $(60 \pm 5 \%)$. ProBNP was the major intracellular form in ventricular myocytes $(58 \pm 4 \%)$. The relative levels of furin mRNA correlated with those of BNP in atrial and ventricular myocytes, and transfection of furin siRNA reduced proBNP processing in both atrial and ventricular myocytes. BNP was the major molecular form in rat plasma $(90 \pm 10 \%)$, whereas proBNP was the major form in human plasma $(72 \pm 8 \%)$. The relative fraction of human BNP in rat myocytes expressing human proBNP was $60 \pm 5 \%$, but increasing the distance between the glycosylation and cleavage sites through mutation, increased the processed fraction correspondingly (Figure 1).

\section{Conclusion}

These results suggest that proBNP is processed into $\mathrm{BNP}$ and $\mathrm{N}$-terminal proBNP intracellularly, most likely by furin in rat. The level of proBNP processing is lower in humans than rats, most likely due to the smaller distance between the $O$-glycosylation and cleavage sites in humans.

\footnotetext{
* Correspondence: nishikim@kuhp.kyoto-u.ac.jp

'Department of Medicine and Clinical Science, Kyoto Univ. Graduate School of Medicine, Japan

Full list of author information is available at the end of the article
} 


\section{FIGURE A, B, C, D, E. Schematic representation of human proBNP and its mutants.}

(A)

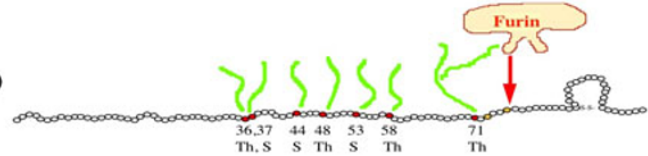

(B)

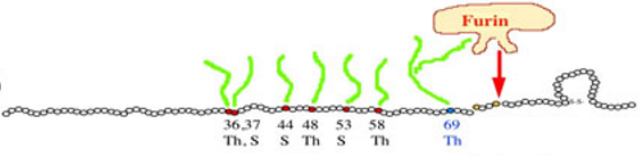

(C)

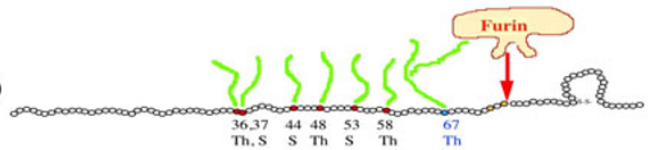

(D)

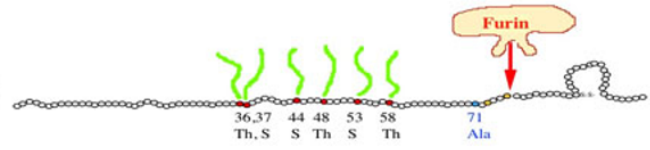

(E)
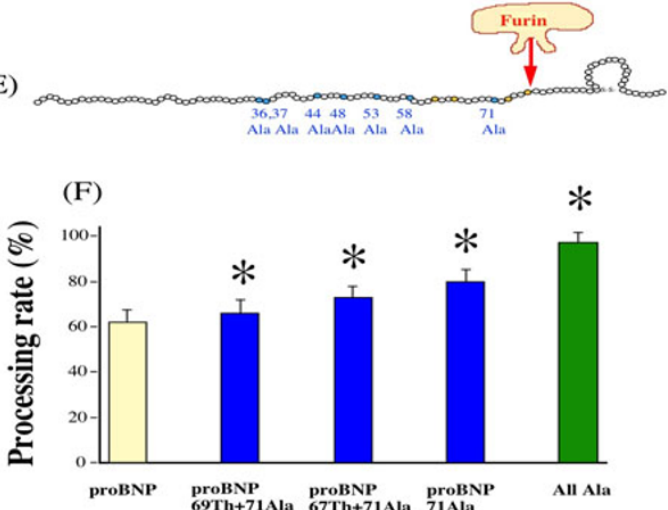

A: wild proBNP

B: L69T/T71A

C: M67T/T71A

D: T71A

E: T36A/S37A/S44A/T48A/ S53A/T58A/T71A

F. Processed fractions (\%) of human proBNP and its mutants.

Figure 1

\section{Authors' details}

'Department of Medicine and Clinical Science, Kyoto Univ. Graduate School of Medicine, Japan. ${ }^{2}$ Department of Hypertension and Cardiorenal Medicine, Dokkyo Medical University, Japan. ${ }^{3}$ Department of Laboratory Medicine Dokkyo Medical University, Japan. ${ }^{4}$ Research Institute National Cardiovascular Research Center, Japan. ${ }^{5}$ Department of Cardiology, Fujii Hospital, Japan.

Published: 29 August 2013

\section{References}

1. Nishikimi T, Kuwahara K, Nakagawa Y, Kangawa K, Minamino N, Nakao K: Complexity of molecular forms of B-type natriuretic peptide in heart failure. Heart 2013, 99:677-679.

2. Nishikimi T, Ikeda M, Takeda Y, Ishimitsu T, Shibasaki I, Fukuda H, Kinoshita H, Nakagawa Y, Kuwahara K, Nakao K: The effect of glycosylation on plasma $\mathrm{N}$ - terminal proBNP-76 levels in patients with heart or renal failure. Heart 2012, 98:152-161.

doi:10.1186/2050-6511-14-S1-P50

Cite this article as: Nishikimi et al:: O-glycosylated pro-B-type natriuretic peptide is cleaved intracellularly by furin in ventricular and atrial myocytes: importance of the distance between the O-glycosylation and cleavage sites. BMC Pharmacology and Toxicology 2013 14(Suppl 1): P50.

Submit your next manuscript to BioMed Central and take full advantage of:

- Convenient online submission

- Thorough peer review

- No space constraints or color figure charges

- Immediate publication on acceptance

- Inclusion in PubMed, CAS, Scopus and Google Scholar

- Research which is freely available for redistribution 